\title{
Can body size predict infant energy requirements?
}

\author{
Jonathan C K Wells, Peter S W Davies
}

\begin{abstract}
Traditionally, infant energy requirements have been predicted from body size or age, whereas in older children and adults, physical activity is also taken into account. However, the extent to which body size determines energy use in individual infants has not been considered. Data on 232 measurements of total energy expenditure obtained in 124 infants aged $\mathbf{1 . 5}$ to 12 months were used to assess the relation between body size and energy use in individuals. Age, weight, and fat free mass consistently predicted total energy expenditure with an error of $21-23 \%$. This contrasts greatly with the error of $10 \%$ with which infant basal metabolism can be predicted from anthropometry. Body size is a poor index of the total energy requirements of individual infants, and predictive equations generated from data on healthy infants will be inappropriate for disease states where physical activity or growth is altered.

(Arch Dis Child 1999;81:429-430)
\end{abstract}

Keywords: energy metabolism; total energy expenditure; fat free mass; body composition

The 1985 WHO guidelines on energy requirements state that once body size, activity level, and growth rate have been accounted for, there is only one level of energy intake at which energy balance can be achieved. ${ }^{1}$ This then becomes the individual's requirement for energy, and is expected to vary minimally providing the above factors remain unchanged. Energy requirements of individual adults are therefore estimated from basal metabolic rate (BMR), which represents body size, and physical activity level (PAL) ${ }^{1}$ calculated as total energy expenditure (TEE) divided by BMR. Adult PAL values can be estimated from activity diaries using data gathered from many studies.

However, requirements of infants are predicted only from body weight, ${ }^{2}$ or age,${ }^{1}$ which acts as a proxy for ideal weight at a given time point. This approach assumes that body size is the principal determinant of energy requirements, and ignores variation between individuals caused by other factors, including physical activity. BMR is indeed closely related to body size in infancy, ${ }^{3}$ as in adults, but whether the same is true for TEE has not been considered.

Recommendations for energy requirements were originally based on measurements of food intake in all age groups. In 1985, the WHO advised that because observed food intakes might reflect parental feeding habits rather than infant energy use, recommendations should be based instead on measurements of TEE. ${ }^{1}$ This approach, using TEE measurement by the doubly labelled water method combined with estimated requirements for growth, has led to revision of the recommendations for energy intake in infancy. ${ }^{4}$ However, the extent to which body size does indeed determine an individual infant's energy use in early life has not been investigated.

We have collected TEE data, using the doubly labelled water method, from infants throughout the 1 st year of life. TEE comprises most energy requirements, including the energy cost of physical activity and the energy used to synthesise new tissue, but not energy stored in new tissue. The measurement is made over seven days and provides an average daily TEE value. Our data, comprising 232 doubly labelled water measurements and described in detail previously, ${ }^{5}$ allows us to evaluate the extent to which body size or age explain TEE variation in this age group.

\section{Methods}

Measurements were made on 49, 92, 37, 36, and 18 infants at $1.5,3,6,9$, and 12 months, respectively, giving a total of 232 measurements on 124 infants. Body size in kg was represented either by weight, or by fat free mass obtained from total body water, as described previously. ${ }^{5}$ Fat free mass is the proportion of the body that uses energy and is a more appropriate index of body size for predicting TEE; however, usually only the weight is available to clinicians and general practitioners. TEE in megaJoules (MJ) was regressed separately on the two indices of size, and on age (days postpartum). Natural logarithmic values for age, size, and TEE were used to express the error as a per cent of the predicted value.

Because the dataset included 37 infants studied at more than one time point, a second analysis was performed using only one TEE measurement from each of the 124 infants. The revised numbers were $22,42,21,22$, and 18 infants at $1.5,3,6,9$, and 12 months, respectively, with care taken to preserve the same proportion of boys at each time point as far as possible.

\section{Results}

There was a slight excess of girls at most time points; there were $41 \%, 48 \%, 35 \%, 36 \%$, and $55 \%$ of boys at $1.5,3,6,9$, and 12 months, respectively. Before 6 months, infants were either formula fed or breast fed, and in each case intakes of complementary foods were minimal; $41 \%$ and $45 \%$ were breast fed at 1.5 and 3 months, respectively. In the further analysis of 124 infants, there were $45 \%, 48 \%$, 
Table 1 Predicting log TEE from log weight, log FFM, or log age using 232 measurements obtained in 124 infants

\begin{tabular}{llllll}
\hline Predictors & Coefficient & $S E$ & p Value & Error (\%) & $R^{2}(\%)$ \\
\hline Constant & -1.49 & 0.10 & $<0.0001$ & 23.5 & 66.3 \\
Log weight (kg) & 1.16 & 0.05 & $<0.0001$ & & \\
Constant & -1.45 & 0.10 & $<0.0001$ & 23.1 & 67.3 \\
Log FFM (kg) & 1.33 & 0.06 & $<0.0001$ & & \\
Constant & -1.32 & 0.09 & $<0.0001$ & 23.3 & 66.7 \\
Log age (days) & 0.43 & 0.02 & $<0.0001$ & &
\end{tabular}

FFM, fat free mass; TEE, total energy expenditure.

Table 2 Predicting log TEE from log weight, log FFM, or log age using only one measurement for each subject in 124 infants

\begin{tabular}{llllll}
\hline Predictors & Coefficient & $S E$ & $p$ Value & Error (\%) & $R^{2}(\%)$ \\
\hline Constant & -1.31 & 0.13 & $<0.0001$ & 22.8 & 67.2 \\
Log weight (kg) & 1.07 & 0.07 & $<0.0001$ & & 68.0 \\
Constant & -1.24 & 0.13 & $<0.0001$ & 22.6 & 71.8 \\
Log FFM (kg) & 1.22 & 0.08 & $<0.0001$ & & \\
Constant & -1.27 & 0.12 & $<0.0001$ & 21.1 & \\
Log age (days) & 0.42 & 0.02 & $<0.0001$ & & \\
\hline
\end{tabular}

FFM, fat free mass; TEE, total energy expenditure.

$33 \%, 45 \%$, and $55 \%$ of boys at $1.5,3,6,9$, and 12 months, respectively, and $41 \%$ and $50 \%$ of infants were breast fed at 1.5 and 3 months, respectively.

There was a negligible difference between variables in the success of predicting TEE. Body size, represented crudely by weight or age, or more appropriately by fat free mass, predicted TEE with an error of approximately $23 \%$ in the entire sample (table 1). There was no improvement in predictive error when the sexes were considered separately, and neither did the addition of feeding mode improve the model (data not shown). For the 124 infants where only one data point was included for each infant, the error was negligibly reduced to $21-22 \%$ (table 2 ).

\section{Discussion}

The consistent predictive error of $21-23 \%$ with which TEE can be predicted from body size or age contrasts greatly with the error of $10 \%$ with which infant BMR can be predicted from anthropometry. ${ }^{3}$ This is reflected in a higher coefficient of variation for TEE (19\%) than for BMR (10\%), indicating that TEE shows greater variation between individuals. ${ }^{5}$ The confidence limits of a predicted TEE value are $\pm 46 \%$, which is clearly very poor, and the range of TEE for a given body weight is more than twofold.

The finding that log weight and log fat free mass predicted log TEE with equal error shows that weight is no worse an index of body size than fat free mass for predicting TEE in this age group. Thus, the problem in this age group is not that weight is a poor index of the proportion of body weight that uses energy. Equally, age appears to act as a satisfactory proxy for body size in this situation. However, body size alone is a poor predictor of infant TEE per se, and physical activity and growth rate would need to be taken into account to reduce predictive error in healthy infants. We have shown in a subset of the infants described above that measures of infant behaviour, collected using a 24 hour activity diary, substantially improve the success with which TEE can be predicted in individuals. ${ }^{6}$

Feeding mode is an additional factor that is known to influence TEE in early infancy, with breast fed infants having moderately lower average values than formula fed infants after taking body size into account. ${ }^{4}$ However, studies indicate that the feeding mode tends to influence basal metabolism, ${ }^{7}$ rather than the energy cost of activity, ${ }^{578}$ and its influence is likely to be minimised once complementary feeding becomes established. The inclusion of feeding mode did not improve our model, presumably because within each diet group between-subject variation in physical activity remains high. ${ }^{89}$

Small for gestational age infants comprise a further group in whom the relation between body size and energy use is different from normal in early life. ${ }^{10}$ TEE $/ \mathrm{kg}$ of fat free mass is raised compared with infants born at an appropriate weight for gestational age, presumably because of differences between the groups in body composition. ${ }^{10}$ Alterations of body composition, influencing the ratio of organ to muscle tissues, which have different energy requirements per unit mass, will inevitably distort the general relation between weight, fat free mass, or age, and TEE.

\section{Conclusion}

The ability of body size to predict TEE, and by inference energy requirements, is limited in individual infants. Clinicians should be aware of the poor relation between body size and energy requirements in early life even in healthy infants, and that prediction equations derived from healthy infants might be inappropriate for disease states where physical activity, growth rates, or body composition are different from normal. Data on the influence of different diseases and treatment regimens on energy use and body composition are required to improve the ability to predict requirements in individual hospitalised infants.

We are very grateful to Professor T Cole of the Institute of Child Health for his expert statistical advice.

1 Energy and protein requirements. Report of a joint FAO/WHO/UNU expert consultation. World Health Organ Tech Rep Ser 1985;724:1-206.

2 Department of Health. Dietary reference values for food energy and nutrients for the United Kingdom. Report on health and and nutrients for the United Kingdom. Repo

3 Butte NF. Basal metabolism of infants. In: Schürch B, Scrimshaw NS, eds. Activity, energy expenditure and energy requirements of infants and children. Lausanne: IDECG, 1990:117-36.

4 Butte NF. Energy requirements of infants. Eur 7 Clin Nutr 1996;50 (suppl 1):S24-36.

5 Wells JCK, Davies PSW. Estimation of the energy cost of physical activity in infancy. Arch Dis Child 1998;78:131-6.

6 Wells JCK, Hinds A, Davies PSW. Free-living energy expenditure and behaviour in late infancy. Arch Dis Child 1997;76:490-4.

7 Butte NF, Wong WW, Ferlic L, O'Brian Smith E, Klein PD, Garza C. Energy expenditure and deposition of breast-fed Pediatr Res and formula-fed infa

8 Wells JCK, Davies PSW. Energy cost of activity in twelve week old infants. Am f Hum Biol 1995;7:85-92.

9 Wells JCK, Davies PSW. Diet and behavioural activity in 12-week-old infants. Ann Hum Biol 1995;22:207-15.

10 Davies PSW, Clough H, Bishop NJ, Lucas A, Cole JJ, Cole TJ. Total energy expenditure in small for gestational age infants. Arch Dis Child Fetal Neonatal Ed 1996;75:F46-8. 